\title{
NOTAS
}

\section{Imaginación social para la justicia, misión de la universidad'}

\author{
José María Margenat Peralta S.I. ${ }^{2}$
}

Resumen: A partir del concepto "imaginación social" utilizado por Pablo Vly de la tensión virtuosa entre mediocritas y radicalidad se reflexiona en tres tiempos: la propuesta de una nueva síntesis humanista (momento hermenéutico), la necesidad de indagar los criterios y lugares sociales de la misión universitaria (momento epistemológico) y la unidad intrínseca entre formación ética y compromiso por la justicia en un proyecto de educación humanista (momento ético-político). El autor concluye con la propuesta de una triple cuenta de resultados: inclusividad sostenible, calidad y solidarismo humanista integral.

Palabras clave: imaginación social, nueva síntesis humanista, triple cuenta de resultados.

\section{A social imagination for justice, a mission of the university}

Abstract: From the concept "social imagination" used by Paul VI and the virtuous tension between mediocritas and radicality, author reflects in three stages: the proposal for a new humanistic synthesis (hermeneutical moment), the need to investigate the criteria and social venues university mission (epistemological moment) and the intrinsic unity

\section{L'imagination sociale pour la justice, mission de l'université}

Résumé: Du concept «imaginaire social» utilisé par Paul VI et la tension vertueuse entre mediocritas et radicalité reflète en trois étapes: la proposition d'une nouvelle synthèse humaniste (moment herméneutique), la nécessité d'enquêter sur les critères et lieux sociaux mission universitaire (moment épistémologique) etl'unité intrinsèque entre la

\footnotetext{
' El texto reproduce con ligeras variaciones la comunicación oral "La misión de la universidad en el trabajo por la justicia social" en la mesa sobre "La aportación de la universidad al humanismo" (3-VII-2015) en el Segundo simposio de Pensamiento Social Cristiano de UNIJES, celebrado en Barcelona, en el IQS.

2 Profesor de Historia de la Filosofía Política, Departamento de Humanidades y Filosofía, Universidad Loyola Andalucía. margenat@uloyola.es
} 
between ethics training and commitment to justice in a liberal arts education project (ethical-political moment). The author concludes with the proposal of a triple bottom line: inclusivity and sustainability, quality and comprehensive humanist solidarism.

Keywords: Social imagination, new humanistic synthesis, triple bottom line. formation etl'engagementéthique à la justice dans un projet d'éducation d'arts libéraux (moment éthico-politique). L'auteur conclut avec la proposition d'un triple bottom line: inclusivité intégrale, qualité et le solidarisme humaniste globale.

Mots clé: L'imagination sociale, nouvelle synthèse humaniste, triple bottom line.

Recibida: 16 de enero de 2016. 
Jamás en cualquier otra época había sido tan explícito el llamamiento a la imaginación social. El surgir de la civilización urbana que acompaña,... ino es, en realidad, un verdadero desafío lanzado a la sabiduría del hombre, a su capacidad de organización, a su imaginación prospectiva? Es necesario consagrar a ella esfuerzos de invención y de capitales.... Si el hombre se deja desbordar y no prevé a tiempo la emergencia de los nuevos problemas sociales, éstos se harán demasiado graves como para que se pueda esperar una solución pacífica. (Pablo Vl, Octogesima adveniens, cfr. nn. 10 y 19, 1971).

\section{Una tensión como punto de partida}

La vida virtuosa, leemos en la Ética a Nicómaco, se ensaya necesariamente en

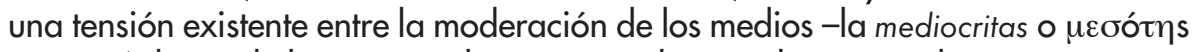
(mesótês), lugar de la virtus en el término medio en relación con los extremos y en relación con las propias capacidades-y la radicalidad constitutiva de la virtud, que se traduce en el alumbramiento del potencial interior de cada persona por la acción mayéutica, la a veces llamada a veces excelencia, que nosotros, sirviéndonos del vocablo de la tradición ignaciana, preferiríamos llamar simplemente "tensión por el magis". Esta radicalidad es doble: por lo que implica de enraizamiento y por lo que señala de extremosidad tensional. En la medida en que se funda en las posibilidades reales que anidan en el interior de la persona, donde habita la verdad, ${ }^{1}$ es radical porque se enraíza en lo interior profundo; pero en la medida en que dirige su desarrollo a las mayores posibilidades, es también radical porque abre horizontes inéditos de humanización, presentes en el homo absconditus en quien anida toda esperanza.

En 1547 el virrey español de Sicilia pidió que los jesuitas establecieran un colegio para seglares en Mesina. "¿Para qué?", se le preguntó. "Para reformar la isla", contestó. Según cuentan, Ignacio, cuando esos hombres partieron para Mesina, les dijo: "Si vivimos diez años más, veremos grandes cosas". ${ }^{2}$ Desde el comienzo, la educación jesuita se propuso la formación de buenos ciudadanos y la contribución a la existencia de buenas ciudades; desde el principio (Mesina fue, sensu stricto, el primer modelo de colegio de la Compañía) se propuso para "reformar" la sociedad; desde el principio la educación jesuita ha sido una educación cívica

\footnotetext{
1 Cf. Agustín de Hipona, De magistro 11:88 y De vera religione 39:72, "Nolite foras ire, in teipsum reddi; in interiore homine habitat veritas"; alguna versión lee: "in interiore hominis", pero preferimos la primera.
}

${ }^{2}$ Cf. J. J. Romero Rodríguez (2007) "Misión de una Universidad jesuita: retos y líneas de futuro": Revista de Fomento Social 393-418 (418). 
enraizada en la tradición humanista italiana, "recibida" junto a otras fuentes inspiradoras en la ratio studiorum. ${ }^{3}$ Ya en el colegio de Mesina cuyo primer rector fue el jesuita Jeroni Nadal se tenía por obligatoria la lectura humanista y formativa de la Ética a Nicómaco. ${ }^{4}$

La tensión descrita necesita y exige el discernimiento como juicio práxico sobre las posibilidades de futuro de este "mundo" (de este orden cósmico, alternativo al caos presente), desde la paciencia de Dios y la prudencia humana (sofrosyne). No hay trabajo por la justicia social sin paciencia y sin prudencia. Entre patientia Dei y prudentia humana, entre moderación y radicalidad, queda abierto un espacio para el discernimiento de los medios, de manera que, considerando siempre a las mujeres y a los hombres, a las personas, como fines, nunca como medios ${ }^{5}$, nos hagamos la pregunta, también ésta radical: ¿qué justicia debe brotar y crecer en la sociedad para que esas personas puedan vivir una existencia digna de hermanos, la fraternidad que proviene de su condición humana creatural? La tensión de esta pregunta se articula en tres momentos: el hermenéutico, el epistemológico y el ético-político.

\section{El momento hermenéutico}

Puesto que es más difícil que antes "sintetizar las varias disciplinas y ramas del saber, como afirma la Constitución Gaudium et spes (n. 61), al haber disminuido "la capacidad para captar y armonizar orgánicamente" el saber, ya que cada vez se desdibuja más la imagen del hombre universal. Esta estructura de la persona la describía el Concilio cuyo recuerdo hacemos, con cuatro palabras: inteligencia, voluntad, conciencia y fraternidad. ¿̇Ha cambiado el diagnóstico? Quizá podríamos subrayar que, cincuenta años después, esa capacidad de síntesis sigue disminuyendo, y la persona integral es menos visible en una sociedad líquida, en una deconstrucción permanente en fragmentos, en la era del vacío y del imperio

\footnotetext{
${ }^{3}$ Cf. J. M. MARgenat (2010) Competentes, conscientes, compasivos y comprometidos. La educación de los jesuitas, Madrid, PPC, así como los estudios, entre otros, de Miquel Batllori, Delio Cantimori, Federico Chabod, Gabriel Codina y Eugenio Garin.
}

${ }^{4}$ Cf. J. M. Margenat (2016) "El sistema educativo de los primeros jesuitas", aceptado para su publicación en Arbor 192.

${ }^{5}$ Kant escribía: "El ser racional debe considerarse siempre como legislador en un reino de los fines posibles por la libertad" (Fundamentación de la Metafísica de las Costumbres, cap. 2; 4, 434 de la edición de la Academia prusiana). 
de lo efímero, por usar las expresiones de pensadores actuales, tan distintos entre ellos como Z. Bauman, N. Elias o G. Lipovetsky.

La persona a la que nos dirigimos, también educativa y universitariamente, ha sido sanada desde la resurrección de los tiempos finales, anticipada en Cristo, y vive atraída por esa resurrección, en la espera de la Pascua que adviene ahora en el tiempo humano, como anticipación virtual y virtuosa de un acontecimiento real. La justicia de Dios se ha anticipado ya del todo y la justicia humana participa internamente de esa justicia. Esta es la condición de posibilidad de la persona integral a la que la universidad quiere ofrecer una nueva síntesis humanista.

Poner en juego estas posibilidades para la educación de las personas, "para el pleno desarrollo cultural (Gaudium et spes n.__) no puede hacerse si no se cuida la pregunta a fondo por el sentido de la cultura y de la ciencia para la persona humana.

En esta pregunta a fondo se sitúan la educación, la investigación y la trasferencia universitarias. Si no nos hacemos la pregunta a fondo, no estamos siendo universidades jesuitas. Si las universidades jesuitas no asumen esta pregunta a fondo, mejor que no existan, porque pensando universitaria y cristianamente ésta es la única razón de su existencia. ${ }^{6}$

Hoy las cosas no han cambiado a mejor, más bien han empeorado por la progresiva fragmentación de los saberes. Hoy se requieren nuevos esfuerzos de comprensión unitaria, de hermeneia y de nueva síntesis humanista, como afirma la encíclica de Benito XVI Caritas in veritate (n. 21). La universidad católica es el lugar natural para trabajar por esa síntesis humanista integral7, por la justicia social. Por eso pensamos que la institución universitaria es un campo privilegiado para la promoción de la justicia en el largo plazo, al menos en tres ámbitos: ${ }^{8}$

${ }^{6}$ Cf. S. ZAMAGNI (2013), "A identidades e a missâo de uma universidade católica na atualidade": Cadernos ihu ideias 11, 3-11, n 185, Universidade Unisinos (Brasil) Instituto Humanitas Unisinos.

7 Cf. J. M. Margenat (2009) "La fraternidad, camino para la familia humana": Revista de Fomento Social 64, 725-734 y J. M. Margenat (2014) "Caridad y verdad", en J. Sols (2014) Pensamiento social cristiano abierto al siglo XXI. A partir de la encíclica Caritas in veritate, Santander, Sal Terrae, 45-72; en ambos textos me he referido a esta nueva síntesis humanista que hay que promover en las universidades jesuitas, entendida como una expresión de aquel humanismo integral o nuevo humanismo teorizado por Jacques Maritain en los años 30 y propuesto por Pablo VI, Benito XVI y Francisco en diferentes documentos sociales. La formación de pensadores de reflexión profunda parece ser una de las misiones que dan sentido a las universidades jesuitas. Si logramos esa reflexión profunda unida a la imaginación social habremos contribuido a cambiar rectamente este mundo.

${ }^{8}$ Según podemos leer en el Documento "La Promoción de la Justicia en las Universidades de la Com- 
a) La educación universitaria formativa según el conocido como paradigma Ledesma-Kolvenbach: lo que los estudiantes lleguen a ser;

b) La investigación capaz de desentrañar las causas estructurales que dan lugar a la injusticiay de sugerir propuestas generadoras de mejoras significativas; $y$, por último,

c) La institucionalidad universitaria como modelo de referencia en su entorno, en relación con la justicia; es decir, la cultura interna de cada institución universitaria, incluida en ella la responsabilidad social universitaria, así como el modo de enfrentar la realidad.

Por otra parte, la universidad católica ha de proponer unas visión de las humanidades que integre la memoria humana del pasado y la prospectiva generadora de posibilidades de futuro, todo ello con el desarrollo de una razón en tres dimensiones: una razón anamnética, crítica de toda memoria, "cautiva y/o cautivada", servil a los intereses de pocos o simplemente reproductora de un desorden impuesto; una razón profética anticipadora de experiencias de justicia realizada, y una razón mayéutica creadora de justicia.

En esto consiste esta nueva síntesis humanista, que reconcilia la fontalidad de Dios con la fraternidad entre los hombres y con la radicación en la tierra, casa común. De esta triple reconciliación habló la trigésimo quinta Congregación general de la Compañía de Jesús (2008) como una de las claves de actuales de comprensión de la misión. ${ }^{9}$

\section{El momento epistemológico}

La crisis no simplemente ha ocurrido, sino que puede ser un lugar epistemológico, una "ocasión", escribía en 2009 Benito XVI, para "discernir y proyectar de un modo nuevo una nueva síntesis humanista (...) desde la imaginación social" (Caritas in veritate, n._.).

pañía", publicado en otoño de 2014 por el Secretariado pata la Justicia Social y la Ecología. Cfr P. Álvarez, editor (2014) Promotio lustititae n. 116, 1-55; es cierto que consta como "no oficial" y "especial", pero apareció con una carta de presentación o prefacio de Michael J. GARANZINI, Secretario de la Compañía de Jesús para la Educación superior y entonces Rector de la Universidad Loyola de Chicago y ahora su Canciller.

9En el decreto 3, "Desafíos para nuestra misión hoy", se dice: "Llamados a establecer relaciones justas. Misión de reconciliación" (nn. 12-36). 
El momento epistemológico de la misión universitaria formula aquellas preguntas inquietantes que permiten cuestionar las evidencias de nuestro conocimiento y de nuestros objetivos. La universidad debe contribuir al cambio social desde el trabajo por la justicia, formulando preguntas "molestas" sobre lo que sólo es "empíricamente" constatable o numéricamente expresable, previa reducción epistemológica.

La universidad, no es, o no debe ser, un lugar para simples evidencias empíricas o para la reproducción social del des-orden (la desigualdad estructural, donde un polo del imán es el factor causal del alejamiento del otro polo del imán, excluido o prescindible, los más ricos que no son tales casualmente junto a los más pobres, sino que son los más ricos porque expropian a los pobres y a los más pobres; la relación no es casual sino causal). Dicho de otra forma: si 62 personas en todo nuestro mundo tienen tanta riqueza como tres mil seiscientos millones de personas (lo que equivale a la mitad de la humanidad), eso no es ni puede ser casual. Es simplemente resultado causal de una distribución inequitativa de posibilidades que bloquea las capacidades de la gran mayoría de la humanidad. La universidad en general, y "a fortiori" las universidades jesuitas en cuanto sustantivamente son universidades, no pueden estar cerradas, ciegas epistemológicamente, ante esta injusticia que opaca la verdad de Dios (cfr. Carta de san Pablo a los Romanos 1:18 "...toda impiedad e injusticia que aprisionan la verdad en la injusticia"), ante esta verdad que pone en evidencia dónde se realiza la verdadera caridad, el amor en la verdad (caritas in veritate). Es una cuestión esencialmente epistemológica, no primariamente ética.

Los aspectos de la crisis y sus soluciones, así como la posibilidad de un nuevo desarrollo futuro, están cada vez más interrelacionados y se implican recíprocamente, requieren nuevos esfuerzos de comprensión unitaria y una nueva síntesis humanista escribió Benito XVI (Caritas in Veritate, n. 21). No hay posibilidad de responder a los retos del desarrollo que nos plantea esta injusticia estructural si no hacemos, como pensadores de reflexión profunda, como comunidad intelectual en las universidades, aquellos esfuerzos de comprensión unitaria y de la nueva síntesis humanista que este momento requiere.

Por esto proponemos como núcleo central de nuestra comunicación un esfuerzo para introducir en las universidades jesuitas un índice de evaluación de calidad que se centre en el desarrollo de la competencia o capacidad de imaginación social aplicada a la lucha contra la injusticia, la social y la ecológica. El momento epistemológico debe alejarse de la simple o cómplice reproducción social de la injusticia, para adentrarse en el terreno de la imaginación social de la justicia, a la que se refirió Pablo VI: 
Jamás en cualquier otra época había sido tan explícito el llamamiento a la imaginación social. Es necesario consagrar a ella esfuerzos de inversión y de capitales tan importantes como los invertidos en armamentos o para las conquistas tecnológicas. ${ }^{10}$

¿Existe una misión de la universidad para ejercer esta imaginación social por la justicia? Creemos que sí, que la universidad debe incorporar a su proyecto la formación, la investigación y la trasferencia sobre esta capacidad de imaginación social.

Pero, surge otra pregunta esencial: ¿̇la buscan o la desean nuestros estudiantes? Según el estudio llevado a cabo por la Federación Internacional de Universidades Católicas (FIUC) entre 2011 y $2014^{11}$, cuando se les preguntó a aquéllos sobre las necesidades más importantes en su vida, sólo el 1,2\% de los estudiantes en universidades católicas decía "interesarse por la política" como lo más importante para su formación y sólo el $10 \%$ de los estudiantes consideraba que el trabajo ideal al que aspiraban debería incluir "propósitos altruistas". Si bien el 82,7 de los estudiantes encuestados en más de 200 instituciones de todo el mundo, creía que la democracia es la mejor forma de gobierno, la mayoría de los estudiantes se mantenía a distancia del "espectáculo" del mundo político en una corta distancia "reducida" a la familia, los amigos y los estudios. Mientras que para el 62,4 de los estudiantes lo más importante es conseguir un buen trabajo-y parece que en esto las universidades católicas destacan-, "fundar la propia familia" (45,4\%) y "ganar mucho dinero" (30\%), de manera llamativa contrasta que implicarse en movimientos pro-derechos humanos o similares sólo es importante para el 4,8\%; tan sólo quedaba por debajo de esta necesidad percibida la de "profundizar en la fe en grupo" (el 2,6\%). Estos universitarios dicen estar felices en sus vidas (la nota que ponen es de 5 sobre 6, muy alta) y no ser pesimistas como hace unos años, pero no les preocupan las grandes cuestiones con que se enfrenta la humanidad actual, sino las de "corta distancia". La pregunta es obvia, aunque podrá ser tan hiriente como impertinente. ¿̇Para qué creamos o mantenemos universidades católicas?

Si leemos unas líneas más del citado texto de papa Montini llegamos a la advertencia, jurgente hace 44 años!: Si la humanidad se deja desbordar y no prevé a tiempo la emergencia de los nuevos problemas sociales, éstos se harán demasiado graves como para que se pueda esperar una solución pacíica. ¿Cuál tiene que ser la diferencia de renta entre Pozuelo (Madrid) y Sanlúcar de Barrameda (Cádiz)

10 PABLO VI (1971) Carta apostólica Octogesima adveniens, n. 19.

${ }^{11}$ Cf. el estudio de R. Aparicio, A. Tornos y D. Rodríguez AzCárate (2015) "Jóvenes en las universidades católicas. Encuesta internacional 2014": Concilium 50, 227-247, estudio a partir del Informe "Las culturas de los jóvenes en las universidades católicas" de la FIUC de 2014, realizado a partir de más de 200 universidades y publicado en inglés, castellano y francés. 
para que sean "un problema demasiado grave"? En el estudio, recientemente hecho público por el INE, que recoge indicadores de 109 ciudades españolas se destaca que la ratio que se establece entre los extremos es de 6,82 en lo que concierne a la renta y de 3,76 en lo que se refiere al desempleo. ${ }^{12}$

\section{El momento ético-político}

Estamos convocados a ejercer la imaginación social y creativa desde la profundidad del aprendizaje, la docencia, la investigación y la trasferencia. El mundo no puede prescindir de la levadura de la universidades católicas para responder a los desafíos de la injusticia, y nosotros, universidades católicas, no tenemos razón de ser ni sentido, si no revisamos toda nuestra acción desde el compromiso y la acción por la justicia.

La reflexión ética y el compromiso por la justicia no son un añadido de calidad, ni una simple consecuencia lógica de un fundamento ajeno, sino que atraviesan y entrañan permanentemente la acción universitaria, toda la acción universitaria y cívica. La reflexión ética no es un sector, un área, una señal de nuestra identidad y misión, sino el nervio que recorre toda la identidad y la misión. Éstas no son dos polos, sino una sola realidad, aunque las distingamos para unirlas en la praxis. Distinguimos identidad y misión para unirlas en su realización: la identidad es la misión, y ésta es la identidad. No puede ser de otra manera, pues, como sabemos, desde Gaudium et spes la naturaleza de la Iglesia -su ser- se identifica con su misión -evangelizar.

De la misma manera, afirmamos que la ética no es sólo personal o sólo social; no hay dicotomías. Sólo logrando un compromiso virtuoso en nuestro ser social, nuestro ser humano, llegamos a ponernos en el camino de la plena humanización de nuestra realidad humana personal, a vivirnos como realidad vocacionada.

Pero el trabajo por la justicia desde la universidad es un ejercicio de razón y libertad, de verdadera imaginación social, que no puede reducirse a consignas, por aparentemente éticas que parezcan, ni a ideología, ni menos aún a consideraciones pietistas. La razón y la libertad, la imaginación social, "comparten un potencial revolucionario de enorme fuerza explosiva" ${ }^{13}$ y responden a una ob-ligación, pero no a una necesidad fatídica, sino una obligación en libertad.

12 El País, 1-VII-2015, 21.

${ }^{13}$ BeNITO XVI (2010) Encíclica Spe salvi n. 18. 
Esta obligación responde a las preguntas: "¿qué podemos esperar?" y "¿qué no podemos esperar?". Ciertamente, a partir de la calidad de nuestro análisis de la complejidad de lo real y de su carácter contradictorio e inabarcable, podemos esperar contribuir a una nueva síntesis humanista que proponga líneas de respuesta.

Mientras el progreso material es acumulativo, en los ámbitos de la conciencia ética y de la decisión moral no es posible un incremento de este tipo por el simple hecho de que la libertad humana es siempre nueva y ha de tomar siempre de nuevo las propias decisiones ${ }^{14}$.

Por tanto, la universidad no puede plantearse desde un hacer científico o académicoreproductor, para luego pasar un "control de calidad ética" externo, añadido, que garantizaría simplemente la buena orientación de fondo, sino que ha de plantear desde el comienzo toda su acción orientándose al fin último de la persona. Cuando, en la segunda mitad del siglo XVI, el jesuita castellano Ledesma proponía para la Ratio del Colegio romano las razones del estudio de las humanidades en la república cristiana y en la lglesia, sostenía que aquel estudio era necesario para el recto gobierno de los asuntos públicos y las (buenas) leyes (el trabajo por la justicia, diríamos hoy) pero, de manera integral (una "nueva síntesis humanista", integrada e integradora) de forma que los estudiantes fueran llevados a su fin último, de manera más cómoda y fácil (commodius et facilius) ${ }^{15}$.

Sólo desde la acción podemos entender el compromiso por la justicia. Sólo desde la correcta colocación hermenéutica y epistemológica. La concepción unitaria de la persona en la sociedad, el personalismo comunitario, y el desarrollo desde la práctica van unidos en el trabajo por la justicia. En este trabajo sucede el proceso de humanización y de construcción de la persona, no al revés. No se es primero persona y luego se trabaja por la justicia como consecuencia de la formación recibida, sino que se trabaja por la justicia y desde ese momento se va siendo persona, más persona, más humano. Por eso las actividades de compromiso, el aprendizaje/servicio, las experiencias de solidaridad, no son el corolario, sino el desencadenante del trabajo por la justicia para todos los miembros de la comunidad universitaria. Como expresó tantas veces el padre Kolvenbach, por ejemplo en el conocido discurso de la universidad de Santa Clara (California), este compromiso nace en la práctica y desde la práctica de estudiantes, docentes e investigadores.

\footnotetext{
14 Ibid. n. 24. Cfr. J. A. Senent de Frutos (2015) "Universidades jesuitas al servicio de la transformación social", en En Compañía. Jesuitas-SAFA-Úbeda, 15-32.

15 Cf. J. M. Margenat (2010) cit., pp. 33 y 162 (y nota 63).
} 
El mismo Kolvenbach decía que no era posible prescindir de las universidades para responder a los desafíos de la injusticia ${ }^{16}$.

¿Deberíamos ser evaluados como universidad católica en una imaginable triple cuenta de resultados? ¿¿Deberíamos ser evaluados no sólo en sostenibilidad -entendida como viabilidad económica-, en calidad de producción científico-investigadora y de capacitación profesional de excelencia? Podemos avanzar la pregunta: jacabe una evaluación en imaginación social y creativa en el trabajo por la justicia? Si no queremos que la pregunta por la razón de ser de las universidades católicas sea sólo una retórica impertinente, ¿̇deberíamos entrar a fondo en esta espesura del compromiso y trabajo por la justicia? ${ }^{17}$

Estamos convocados a aquella imaginación social de la que escribió Pablo VI en 1971. Desde ella -imaginación creativa la llamaba Adolfo Nicolás en la Universidad Iberoamericana de México (2010) y desde la profundidad del aprendizaje que acompañan e integran rigor intelectual, reflexión sobre la experiencia de la realidad, es posible "trabajar por construir un más humano, justo, sostenible y lleno de fe".

\section{Conclusión: una triple cuenta de resultados para las universi- dades jesuitas}

Como hemos anticipado, nuestra propuesta es introducir en las universidades jesuitas un índice de evaluación de calidad, a modo de una triple cuenta de resultados, que haya que presentar en los órganos de gobierno de las mismas (patronatos, consejos de dirección, consejos de gobierno, senados académicos,...). En primer lugar, el resultado de la sostenibilidad humana, social, ecológica y económica de la institución universitaria, atendiendo a todos los aspectos incluidos en esa cuádruple

\footnotetext{
${ }^{16}$ Lección inaugural en Universidad San Alberto Hurtado de Chile en P. H. KolvenBACH (2009), El P. Peter-Hans Kolvenbach, S. I. y la educación 1983-2007 (Selección de Escritos), Bogotá, ACODESI, pp. 243-252 (1-V-2006).

${ }^{17}$ El 30 de marzo de 2012 el cardenal Peter K. A. Turkson, presidente del Pontificio Consejo «Justicia y Paz», en un discurso a dos mil empresarios cristianos presentes en el 24 Congreso mundial de UNIAPAC, celebrado en Lyon, Francia, presentó el documento "La Vocación del Líder Empresarial: Una Reflexión", concebido como un vademécum dirigido a los profesores de las universidades y a los empresarios en su compromiso cotidiano para integrar la fe, actividad formativa y actividad laboral. Ciertamente, jhay que entrar en las espesuras!
} 
rejilla, es decir a todos los agentes implicados en su desarrollo (stakeholders): la comunidad humana de personas, la cohesión y la reforma social, el impacto en el medio y finalmente la actividad económica con función social y, por tanto, con viabilidad. En segundo lugar, el resultado de la calidad docente e investigadora, y la trasferencia social de conocimiento generada por las mismas. En tercer lugar, el resultado de la solidaridad humanista integral. ${ }^{18} \mathrm{~A}$ esta última nos hemos referido en la presente comunicación.

El resultado de la solidaridad humanista integral se podrá medir en la medida en que las instituciones hayan formulado en su llamado plan estratégico y en sus declaraciones de identidad y misión, visión y valores, el objetivo de logra que los miembros de la comunidad universitaria, estudiantes, investigadores, administradores y personal de servicios y, por último pero no en último lugar, los profesores hayan avanzado en el desarrollo de la capacidad de imaginación social aplicada a la lucha contra la injusticia, la social y la ecológica. Ésta debería ser misión de todos y cada uno de los miembros de la comunidad universitaria y de ésta en su conjunto. La acción y el compromiso por la justicia se adentran así en el fruto de una sociedad más inclusiva en aspectos esenciales a los que afectan los derechos humanos: inclusividad de género, étnica, social (de clase o de los excluidos), ideológica, etc. Para esta acción y este compromiso hace falta que la comunidad universitaria, y cada uno de sus agentes, haga suya la imaginación social a que nos hemos referido antes. Ésa es la misión de la universidad jesuita, ésa es nuestra confianza, ése es nuestro desafío.

\footnotetext{
${ }^{18}$ En el momento de enviar a imprenta este número se ha publicado un interesante Informe especial titulado "Por una economía global justa. Construir sociedades sostenibles e inclusivas": Promotio lustitiae n. 121, 2016/1.
} 